\title{
A Study on the Solutions of a Multiterm FBVP of Variable Order
}

\author{
Zoubida Bouazza, ${ }^{1}$ Sina Etemad, ${ }^{2}$ Mohammed Said Souid, ${ }^{3}$ Shahram Rezapour $\mathbb{D}^{2,4}$ \\ Francisco Martínez, ${ }^{5}$ and Mohammed K. A. Kaabar $\mathbb{1}^{6}$ \\ ${ }^{1}$ Laboratory of Mathematics, Djillali Liabes University of Sidi Bel-Abbès, Algeria \\ ${ }^{2}$ Department of Mathematics, Azarbaijan Shahid Madani University, Tabriz, Iran \\ ${ }^{3}$ Department of Economic Sciences, University of Tiaret, Algeria \\ ${ }^{4}$ Department of Medical Research, China Medical University Hospital, China Medical University, Taichung, Taiwan \\ ${ }^{5}$ Department of Applied Mathematics and Statistics, Technological University of Cartagena, Cartagena 30203, Spain \\ ${ }^{6}$ Jabalia Camp, United Nations Relief and Works Agency (UNRWA) Palestinian Refugee Camp, Gaza Strip Jabalya, State of Palestine
}

Correspondence should be addressed to Shahram Rezapour; rezapourshahram@yahoo.ca and Mohammed K. A. Kaabar; mohammed.kaabar@wsu.edu

Received 25 March 2021; Accepted 27 April 2021; Published 24 May 2021

Academic Editor: Jiabin Zuo

Copyright (c) 2021 Zoubida Bouazza et al. This is an open access article distributed under the Creative Commons Attribution License, which permits unrestricted use, distribution, and reproduction in any medium, provided the original work is properly cited.

In the present research study, for a given multiterm boundary value problem (BVP) involving the nonlinear fractional differential equation (NnLFDEq) of variable order, the uniqueness-existence properties are analyzed. To arrive at such an aim, we first investigate some specifications of this kind of variable order operator and then derive required criteria confirming the existence of solution. All results in this study are established with the help of two fixed-point theorems and examined by a practical example.

\section{Introduction}

The first historical resource to the invention of fractional derivative (FrDr) was proposed in 1695, when L'Hopital proposed the question about the meaning of $d^{\sigma} y / d x^{\sigma}$ if $\sigma=1 / 2$. The proposed FrDr's definitions are classified into two categories: global nature and local nature. On the one hand, under the global one, the FrDr is defined as integral, Fourier, or Mellin transformations; hence, its nonlocal characteristic is given with a memory. On the other hand, under the local one, FrDr is relied on a local definition through certain incremental ratios. As a result of this classical formulation, fractional calculus ( $\mathrm{FrCa}$ ) has appeared since the time of wellknown mathematicians such as Lacroix, Fourier, Liouville, Euler, Laplace, and Abel until the creation of the first modern fractional definitions of Caputo and Riemann-Liouville.

The FrCa theory is a representation of a powerful tool of mathematical analysis for investigating the integrals and derivatives of arbitrary order, which constitutes the unifying and generalizing element of the integer-order differentiation and $n$-fold integration [1,2]. Studying fractional integrals and derivatives was only devoted to the theoretical mathematical context. However, their applications have been recently seen in multidisciplinary sciences such as theoretical physics, entropy theory, fluid mechanics, biology, and image processing [3-13].

Furthermore, studying both of the theoretical and practical aspects of fractional differential equations (FDEqs) has become a focus of international academic research [14-18]. A recent improvement on this investigation is the consideration of the notion of variable order operators. In this sense, various definitions of fractional operators involving the variable order have been introduced. This type of operators which are dependent on their power-law kernel can describe some hereditary specifications of numerous processes and phenomena $[19,20]$. In general, it is often difficult to find the analytical solution of FDEqs of variable order; therefore, numerical methods for the approximation of FDEqs of variable order are widespread. Regarding the study of solutions' existence to the problems of variable order, we refer to [21- 
24]. On the contrary, a consistent approach with the firstorder precision for the solution of FDEqs of variable order is applied by Coimbra in [25]. Lin et al. [26] discussed the convergence and stability of an explicit approximation related to the diffusion equation of variable order with a nonlinear source term. In [27], Zhuang et al. introduced the implicit and explicit Euler approximations for the nonlinear diffusion-advection equation of variable order.

While several research studies have been performed on investigating the solutions' existence of the fractional constant-order problems, the solutions' existence of the variable-order problems are rarely discussed in literature; we refer to [28-34]. Therefore, investigating this interesting special research topic makes all our results novel and worthy.

Inspired by all previous studies, this work investigates the solutions' existence to the proposed multiterm BVP for NnLFDEqs of variable order in the format

$$
\left\{\begin{array}{l}
\mathfrak{D}_{0^{+}}^{\Omega(\tau)} x(\tau)+\psi\left(\tau, x(\tau), \mathscr{I}_{0^{+}}^{\Omega(\tau)} x(\tau)\right)=0, \quad \tau \in \mathfrak{J}:=[0, K], \\
x(0)=0, \quad x(K)=0,
\end{array}\right.
$$

where $0<K<+\infty, 1<\Omega(\tau) \leq 2, \psi: \mathfrak{\Im} \times \mathbb{R}^{2} \rightarrow \mathbb{R}$ is continuous, and $\mathfrak{D}_{0^{+}}^{\Omega(\tau)}$ and $\mathscr{I}_{0^{+}}^{\Omega(\tau)}$ are the Riemann-Liouville fractional derivative (RLFrD) and integral (RLFrIn) of variableorder $\Omega(\tau)$, respectively. We here try to express some differences of variable-order operators than standard ones and then implement our techniques to confirm the existence property related to the above BVP.

This work is divided into the following sections: some essential notions are presented in Section 2 that will be used later. Two important results are as follows: one is relied on Schauder fixed-point theorem (Schauder-FixPThm), and the other one is relied on the Banach contraction principle (Banach-CoPrp), which are provided in Section 3. In Section 4 , a numerical example is provided to validate and apply our theoretical results.

\section{Essential Preliminaries}

Some essential mathematical notations that will be used later are provided in this section. By means of $\mathscr{C}(\mathfrak{J}, \mathbb{R})$, let us represent the Banach space (Banach-Sp) of continuous mappings from $\mathfrak{\Im}$ into $\mathbb{R}$ via

$$
\|x\|=\sup \{|x(\tau)|: \tau \in \mathfrak{J}\}
$$

Definition 1 (see $[22,35])$. Let $-\infty<q<p<+\infty$, and $\Omega(\tau)$ : [ $q, p] \rightarrow(0,+\infty)$. The left $\Omega(\tau)^{\text {th }}$-RLFrIn of a function $\xi(\tau)$ is expressed by

$$
\mathscr{J}_{q^{+}}^{\Omega(\tau)} \xi(\tau)=\int_{q}^{\tau} \frac{(\tau-\mu)^{\Omega(\mu)-1}}{\Gamma(\Omega(\mu))} \xi(\mu) d \mu, \quad \tau>q
$$

where the gamma function is denoted by $\Gamma(\cdot)$.
Definition 2 (see $[22,35])$. Let $-\infty<q<p<+\infty, m \in \mathbb{N}$ and $\Omega(\tau):[q, p] \rightarrow(m-1, m)$. The left $\Omega(\tau)^{\text {th }}-$ RLFrD of a function $\xi(\tau)$ is expressed by

$$
\begin{aligned}
\mathfrak{D}_{q^{+}}^{\Omega(\tau)} \xi(\tau) & =\left(\frac{d}{d \tau}\right)^{m} \mathscr{I}_{q^{+}}^{m-\Omega(\tau)} \xi(\tau) \\
& =\left(\frac{d}{d \tau}\right)^{m} \int_{q}^{\tau} \frac{(\tau-\mu)^{m-\Omega(\mu)-1}}{\Gamma(m-\Omega(\mu))} \xi(\mu) d \mu, \quad \tau>q .
\end{aligned}
$$

Obviously, if the order $\Omega(\tau)$ is a constant function $\Omega$, then the Riemann-Liouville variable-order fractional derivative (RLVoFrD) (4) and integral (RLVoIn) (3) are the usual RLFrD and RLFrIn, respectively; see [22, 35].

Let us now discuss some essential properties.

Lemma 3 (see [2]). Let $\gamma>0$. Then, the differential equation

$$
\mathfrak{D}_{q}^{\gamma} \xi=0
$$

has a unique solution:

$$
\begin{aligned}
& \xi(\tau)=\lambda_{1}(\tau-q)^{\gamma-1}+\lambda_{2}(\tau-q)^{\gamma-2}+\cdots+\lambda_{m}(\tau-q)^{\gamma-m} \\
& \lambda_{j} \in \mathbb{R}, j=1,2, \cdots, m \text {, here } m-1<\gamma \leq m .
\end{aligned}
$$

Lemma 4 (see [2]). Letting $q>0, \xi \in L(q, p), \mathfrak{D}_{q}^{\gamma} \xi \in L(q, p)$, then

$$
\begin{aligned}
& \begin{aligned}
\mathscr{J}_{q^{+}}^{\gamma} \mathfrak{D}_{q^{+}}^{\gamma} \xi(\tau)= & \xi(\tau)+\lambda_{1}(\tau-q)^{\gamma-1} \\
& +\lambda_{2}(\tau-q)^{\gamma-2}+\cdots+\lambda_{m}(\tau-q)^{\gamma-m},
\end{aligned} \\
& \lambda_{j} \in \mathbb{R}, j=1,2, \cdots, m \text {, here } m-1<\gamma \leq m .
\end{aligned}
$$

Lemma 5 (see [2]). Letting $\gamma>0$, then we obtain

$$
\mathfrak{D}_{q^{+}}^{\gamma} \mathscr{F}_{q^{+}}^{\gamma} \xi(\tau)=\xi(\tau)
$$

Lemma 6 (see [2]). Letting $\gamma, \delta>0$, then we get

$$
\mathscr{I}_{q^{+}}^{\gamma} \mathscr{J}_{q^{+}}^{\delta} \xi(\tau)=\mathscr{I}_{q^{+}}^{\delta} \mathscr{I}_{q^{+}}^{\gamma} \xi(\tau)=\mathscr{I}_{q^{+}}^{\gamma+\delta} \xi(\tau)
$$

Remark 7 (see [30, 32]). Generally, for functions $\Omega(\tau)$ and $v(\tau)$, the semigroup property does not hold, i.e.,

$$
\mathscr{I}_{q^{+}}^{\Omega(\tau)} \mathscr{J}_{q^{+}}^{v(\tau)} \xi(\tau) \neq \mathscr{F}_{q^{+}}^{\Omega(\tau)+v(\tau)} \xi(\tau)
$$

Example 1. Let

$$
\begin{aligned}
& \Omega(\tau)= \begin{cases}2, & \tau \in[0,1], \\
1, & \tau \in] 1,3],\end{cases} \\
& v(\tau)= \begin{cases}1, & \tau \in[0,1], \\
2, & \tau \in] 1,3],\end{cases}
\end{aligned}
$$


and $\xi(\tau)=\tau$ and $\tau \in[0,3]$. Then, we compute

$$
\begin{aligned}
& \mathscr{F}_{0^{+}}^{\Omega(\tau)} \mathcal{F}_{0^{+}}^{v(\tau)} \xi(\tau)= \int_{0}^{1} \frac{(\tau-\mu)^{\Omega(\mu)-1}}{\Gamma(\Omega(\mu))} \int_{0}^{\mu} \frac{(\mu-\omega)^{v(\omega)-1}}{\Gamma(\nu(\omega))} \xi(\omega) d \omega d \mu \\
&+\int_{1}^{\tau} \frac{(\tau-\mu)^{\Omega(\mu)-1}}{\Gamma(\Omega(\mu))} \int_{0}^{\mu} \frac{(\mu-\omega)^{v(\omega)-1}}{\Gamma(v(\omega))} \xi(\omega) d \omega d \mu \\
&= \int_{0}^{1} \frac{(\tau-\mu)^{1}}{\Gamma(2)} \int_{0}^{\mu} \frac{(\mu-\omega)^{0}}{\Gamma(1)} \omega d \omega d \mu+\int_{1}^{\tau} \frac{(\tau-\mu)^{0}}{\Gamma(1)} \\
& \cdot\left[\int_{0}^{1} \frac{(\mu-\omega)^{0}}{\Gamma(1)} \omega d \omega+\int_{1}^{\mu} \frac{(\mu-\omega)^{1}}{\Gamma(2)} \omega d \omega\right] d \mu \\
&= \int_{0}^{1} \frac{(\tau-\mu) \mu^{2}}{2 \Gamma(2)} d \mu+\int_{1}^{\tau} \frac{\mu^{3}}{6}-\frac{\mu}{2}+\frac{5}{6} d \mu, \\
& \mathscr{F}_{0^{+}}^{\Omega(\tau)+v(\tau)} \xi(\tau)=\int_{0}^{\tau} \frac{(\tau-\mu)^{\Omega(\mu)+v(\mu)-1}}{\Gamma(\Omega(\mu)+v(\mu))} \xi(\mu) d \mu .
\end{aligned}
$$

We see that

$$
\begin{aligned}
\left.\mathscr{J}_{0^{+}}^{\Omega(\tau)} \mathscr{J}_{q^{+}}^{v(\tau)} \xi(\tau)\right|_{\tau=2}= & \int_{0}^{1} \frac{(2-\mu) \mu^{2}}{2 \Gamma(2)} d \mu+\int_{1}^{2} \frac{\mu^{3}}{6}-\frac{\mu}{2} \\
& +\frac{5}{6} d \mu=\frac{5}{24}+\frac{17}{24}=\frac{22}{24} \\
\left.\mathscr{J}_{0^{+}}^{\Omega(\tau)+v(\tau)} \xi(\tau)\right|_{\tau=2}= & \int_{0}^{1} \frac{(2-\mu)^{2+1-1}}{\Gamma(2+1)} \mu d \mu \\
& +\int_{1}^{2} \frac{(2-\mu)^{1+2-1}}{\Gamma(1+2)} \mu d \mu \\
= & \frac{11}{24}+\frac{5}{24}=\frac{16}{24} .
\end{aligned}
$$

Therefore, we obtain

$$
\left.\mathscr{J}_{0^{+}}^{\Omega(\tau)} \mathscr{J}_{0^{+}}^{v(\tau)} \xi(\tau)\right|_{\tau=2} \neq\left.\mathscr{I}_{0^{+}}^{\Omega(\tau)+v(\tau)} \xi(\tau)\right|_{\tau=2}
$$

Lemma 8 (see [24]). Let $0 \leq \zeta<1$ and $\Omega: \mathfrak{\Im} \rightarrow(1,2]$ be continuous. Then, for $y \in \mathscr{C}_{\zeta}(\mathfrak{J}, \mathbb{R})=\left\{y(\tau) \in \mathscr{C}(\mathfrak{\Im}, \mathbb{R}), \tau^{\zeta} y(\tau)\right.$ $\left.\in \mathscr{C}(\mathfrak{J}, \mathbb{R}), \tau \in \min _{\tau \in \mathfrak{I}}|\Omega(\tau)|\right\}$, the variable order fractional integral $\mathscr{I}_{0^{+}}^{\Omega(\tau)} y(\tau)$ exists for any points on $\mathfrak{J}$.

Lemma 9 (see [24]). Let $\Omega: \mathfrak{J} \rightarrow(1,2]$ be a continuous function, then $\mathscr{I}_{0^{+}}^{\Omega(\tau)} y(\tau) \in \mathscr{C}(\mathfrak{I}, \mathbb{R})$ for $y \in \mathscr{C}(\mathfrak{I}, \mathbb{R})$.

Definition 10 (see [34]). We say that the set $\square \subset \mathbb{R}$ is a generalized interval if it is either an interval (one of the formats ( $\left.c_{1}, c_{2}\right),\left[c_{1}, c_{2}\right],\left[c_{1}, c_{2}\right)$, or $\left.\left(c_{1}, c_{2}\right]\right)$ or a single-point $\left\{c_{1}\right\}$, or $\varnothing$.

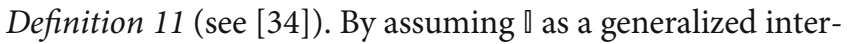
val, a finite set $\mathbb{P}$ consisting of generalized intervals belonging

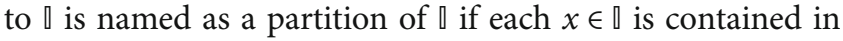
exactly one of the generalized intervals $\mathbb{J}$ in $\mathscr{P}$.

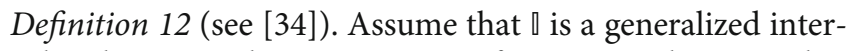
val and suppose that $\rho: \mathbb{\square} \rightarrow \mathbb{R}$ is a function, and assume that $\mathbb{P}$ is a partition of $\square$. Then, $\rho$ is named as a piecewise constant

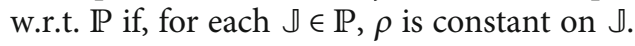

Theorem 13 (see [36] (Schauder-FixPThm)). Assume that $\mathscr{E}$ is a Banach-Sp, and $Q$ as its subset via convexity, closedness, and boundedness and $F: \mathbb{Q} \rightarrow \mathbb{Q}$ via the compactness and continuity. Then, it is found at least a fixed point in $Q$ for $F$.

\section{Existence of Solutions}

All our original main results in this work are discussed in this section. Some assumptions are presented as follows:

(ASP1) Let $m \in \mathbb{N}$ be an integer, $\mathscr{P}=\left\{\mathfrak{\mho}_{1}:=\left[0, K_{1}\right], \mathfrak{\mho}_{2}\right.$ $\left.:=\left(K_{1}, K_{2}\right], \mathfrak{J}_{3}:=\left(K_{2}, K_{3}\right], \cdots \mathfrak{\Im}_{m}:=\left(K_{m-1}, K\right]\right\}$ be a partition of the interval $\mathfrak{\Im}$, and let $\Omega(\tau): \mathfrak{I} \rightarrow(1,2]$ be a piecewise constant function w.r.t. $\mathscr{P}$ as follows:

$$
\Omega(\tau)=\sum_{j=1}^{m} \Omega_{j} \mathbb{1}_{j}(\tau)= \begin{cases}\Omega_{1}, & \text { if } \tau \in \mathfrak{J}_{1}, \\ \Omega_{2}, & \text { if } \tau \in \mathfrak{J}_{2}, \\ \cdot \\ \Omega_{m}, & \text { if } \tau \in \mathfrak{\mho}_{m},\end{cases}
$$

where $1<\Omega_{j} \leq 2$ and $\mathbb{\nabla}_{j}$ is an indicator of intervals $\mathfrak{\Im}_{j}:=($ $\left.K_{j-1}, K_{j}\right], j=1,2, \cdots, m$, such that,

$$
\nabla_{j}(\tau)= \begin{cases}1, & \text { for } \tau \in \mathfrak{\Im}_{j} \\ 0, & \text { for elsewhere }\end{cases}
$$

(ASP2) Let $\tau^{\zeta} \psi: \mathfrak{\Im} \times \mathbb{R} \times \mathbb{R} \rightarrow \mathbb{R}$ be a continuous function $(0 \leq \zeta<1) . \exists$ constants $c_{i}>0, i=1,2,3$ and $0<\sigma<1,0$ $<\eta<1$, such that

$$
\tau^{\zeta}|\psi(\tau, y, z)| \leq c_{1}+c_{2}|y|^{\sigma}+c_{3}|z|^{\eta}
$$

for any $y, z \in \mathbb{R}$ and $\tau \in \mathfrak{J}$.

(ASP3) $\exists$ constants $W, L>0,0 \leq \zeta<1$ such that

$$
\tau^{\zeta}\left|\psi\left(\tau, s_{1}, z_{1}\right)-\psi\left(\tau, s_{2}, z_{2}\right)\right| \leq W\left|s_{1}-s_{2}\right|+L\left|z_{1}-z_{2}\right|,
$$

for any $s_{1}, s_{2}, z_{1}, z_{2} \in \mathbb{R}$ and $\tau \in \mathfrak{J}$.

By $\mathscr{E}_{j}=\mathscr{C}\left(\mathfrak{J}_{j}, \mathbb{R}\right)$, let us represent the Banach-Sp of continuous mappings from $\mathfrak{\Im}_{j}$ into $\mathbb{R}$ via

$$
\|x\|_{\mathscr{E}_{j}}=\sup _{\tau \in \mathfrak{\Im}_{j}}|x(\tau)|
$$

where $j \in\{1,2, \cdots, m\}$. 
To get our original results, let us first perform an essential analysis to our proposed BVP (1). By (4), the equation of the BVP (1) can be written as

$\frac{d^{2}}{d \tau^{2}} \int_{0}^{\tau} \frac{(\tau-\mu)^{1-\Omega(\mu)}}{\Gamma(2-\Omega(\mu))} x(\mu) d \mu+\psi\left(\tau, x(\tau), \mathcal{F}_{0^{+}}^{\Omega(\tau)} x(\tau)\right)=0, \quad \tau \in \mathfrak{J}$.

According to (ASP1), equation (20) on the interval $\mathfrak{\Im}_{j}$, $j=1,2, \cdots, m$ can be written as

$$
\begin{aligned}
& \frac{d^{2}}{d \tau^{2}}\left(\int_{0}^{K_{1}} \frac{(\tau-\mu)^{1-\Omega_{1}}}{\Gamma\left(2-\Omega_{1}\right)} x(\mu) d \mu+\cdots+\int_{K_{j-1}}^{\tau} \frac{(\tau-\mu)^{1-\Omega_{j}}}{\Gamma\left(2-\Omega_{j}\right)} x(\mu) d \mu\right) \\
& \quad+\psi\left(\tau, x(\tau), \mathscr{F}_{0^{+}}^{\Omega_{j}} x(\tau)\right)=0,
\end{aligned}
$$

for $\tau \in \mathfrak{\Im}_{j}$. Let us now define the solution to the BVP (1), which is essential in this research study.

Definition 14. A BVP (1) has a solution, if $\exists$ functions $x_{j}, j$ $=1,2, \cdots, m$, so that $x_{j} \in \mathscr{C}\left(\left[0, K_{j}\right], \mathbb{R}\right)$ that satisfy equation (21) and $x_{j}(0)=0=x_{j}\left(K_{j}\right)$.

From our previous analysis above, (1) can be expressed as equation (20), which can be written on the intervals $\mathfrak{J}_{j}, j \in$ $\{1,2, \cdots, m\}$ as (21). For $0 \leq \tau \leq K_{j-1}$, by taking $x(\tau) \equiv 0$, then (21) is written as follows:

$$
D_{K_{i-1}^{+}}^{u_{i}} x(\tau)+\psi\left(\tau, x(\tau), \mathscr{F}_{K_{i-1}^{+}}^{u_{i}} x(\tau)\right)=0, \quad \tau \in \mathfrak{J}_{i} .
$$

Let us consider the following BVP:

$$
\begin{cases}\mathfrak{D}_{K_{j-1}^{+}}^{\Omega_{j}} x(\tau)+\psi\left(\tau, x(\tau), \mathcal{F}_{K_{j-1}^{+}}^{\Omega_{j}} x(\tau)\right)=0, & \tau \in \mathfrak{J}_{j}, \\ x\left(K_{j-1}\right)=0, & x\left(K_{j}\right)=0 .\end{cases}
$$

For the solutions' existence of problem (23), an auxiliary lemma is needed as follows:

Lemma 15. The function $x \in \mathscr{E}_{j}$ is a solution of problem (23) iff $x$ satisfies the integral equation as follows:

$$
x(\tau)=\int_{K_{j-1}}^{K_{j}} G_{j}(\tau, \mu) \psi\left(\mu, x(\mu), \mathscr{F}_{K_{j-1}^{+}}^{\Omega_{j}} x(\mu)\right) d \mu
$$

where $G_{j}(\tau, \mu)$ is Green's function defined by

$G_{j}(\tau, \mu)=\left\{\begin{array}{l}\frac{1}{\Gamma\left(\Omega_{j}\right)}\left[\left(K_{j}-K_{j-1}\right)^{1-\Omega_{j}}\left(\tau-K_{j-1}\right)^{\Omega_{j}-1}\left(K_{j}-\mu\right)^{\Omega_{j}-1}-(\tau-\mu)^{\Omega_{j}-1}\right], \\ K_{j-1} \leq \mu \leq \tau \leq K_{j}, \\ \frac{1}{\Gamma\left(\Omega_{j}\right)}\left(K_{j}-K_{j-1}\right)^{1-\Omega_{j}}\left(\tau-K_{j-1}\right)^{\Omega_{j}-1}\left(K_{j}-\mu\right)^{\Omega_{j}-1}, \\ K_{j-1} \leq \tau \leq \mu \leq K_{j},\end{array}\right.$

where $j=1,2, \cdots, m$.

Proof. Let $x \in \mathscr{E}_{j}$ be a solution of the BVP (23). Now, let us apply the operator $\mathscr{J}_{K_{j-1}^{+}}^{\Omega_{j}}$ to both sides of the equation of the supposed BVP (23). By Lemma 4, we obtain

$$
\begin{aligned}
x(\tau)= & \lambda_{1}\left(\tau-K_{j-1}\right)^{\Omega_{j}-1}+\lambda_{2}\left(\tau-K_{j-1}\right)^{\Omega_{j}-2} \\
& -\frac{1}{\Gamma\left(\Omega_{j}\right)} \int_{K_{j-1}}^{\tau}(\tau-\mu)^{\Omega_{j}-1} \psi \\
& \cdot\left(\mu, x(\mu), \mathscr{J}_{K_{j-1}^{+}}^{\Omega_{j}} x(\mu)\right) d \mu, \quad \tau \in \mathfrak{\Im}_{j} .
\end{aligned}
$$

By $x\left(K_{j-1}\right)=0$ and the function $\psi$, we obtain $\lambda_{2}=0$. Let $x(\tau)$ satisfies $x\left(K_{j}\right)=0$. Thus, we get $\lambda_{1}=\left(K_{j}-K_{j-1}\right)^{1-\Omega_{j}}$ $\mathscr{\mathcal { F }}_{K_{j-1}^{+}}^{\Omega_{j}} \psi\left(K_{j}, x\left(K_{j}\right), \mathcal{F}_{K_{j-1}^{+}}^{\Omega_{j}} x\left(K_{j}\right)\right)$. Then, we have

$$
\begin{aligned}
x(\tau)= & \left(K_{j}-K_{j-1}\right)^{1-\Omega_{j}}\left(\tau-K_{j-1}\right)^{\Omega_{j-1}} \mathscr{F}_{K_{j-1}^{\Omega_{j}}}^{\Omega_{j}} \psi \\
& \cdot\left(K_{j}, x\left(K_{j}\right), \mathcal{F}_{K_{j-1}^{j}}^{\Omega_{j}} x\left(K_{j}\right)\right)-\mathcal{F}_{K_{j-1}^{+}}^{\Omega_{j}} \psi\left(\tau, x(\tau), \mathcal{F}_{K_{j-1}^{\Omega_{j}}}^{\Omega_{j}} x(\tau)\right), \quad \tau \in \mathfrak{F}_{j},
\end{aligned}
$$

by the continuity of Green's function which implies that

$$
x(\tau)=\int_{K_{j-1}}^{K_{j}} G_{j}(\tau, \mu) \psi\left(\mu, x(\mu), \mathscr{F}_{K_{j-1}^{+}}^{\Omega_{j}} x(\mu)\right) d \mu .
$$

Conversely, let $x \in \mathscr{E}_{j}$ be a solution of integral equation (24); then, by the continuity of function $\tau^{\zeta} \psi$ and Lemma 5, we can easily get that $x$ is the solution of BVP (23).

The following proposition will be needed:

Proposition 16 (see [34]). Let $0 \leq \zeta<1$ and assume that $\tau^{\zeta}$ $\psi: \mathfrak{J} \times \mathbb{R} \times \mathbb{R} \rightarrow \mathbb{R}$ is continuous, $\Omega(\tau): \mathfrak{J} \rightarrow(1,2]$ satisfies (ASP1). Then, Green's function of BVP (23) satisfies the following properties:

(I) $G_{j}(\tau, \mu) \geq 0, \forall K_{j-1} \leq \tau, \mu \leq K_{j}$

(II) $\max _{\tau \in \mathfrak{\Im}_{j}} G_{j}(\tau, \mu)=G_{j}(\mu, \mu), \mu \in \mathfrak{\Im}_{j}$

(III) $G_{j}(\mu, \mu)$ has one unique maximum given by 


$$
\max _{\mu \in \mathfrak{\Im}_{j}} G_{j}(\mu, \mu)=\frac{1}{\Gamma\left(\Omega_{j}\right)}\left(\frac{K_{j}-K_{j-1}}{4}\right)^{\Omega_{j}-1}
$$

where $j=1,2, \cdots, m$.

The first existence result is relied on Theorem 13 .

Theorem 17. Suppose that (ASP1)-(ASP3) hold; then, it is found a solution to the BVP (1) on $\mathfrak{\Im}$.

Proof. Problem (23) can be transformed into a fixed-point problem. Let us construct the following operator:

$$
\mathscr{M}: \mathscr{E}_{j} \rightarrow \mathscr{E}_{j}
$$

formulated by

$$
\mathscr{M} x(\tau)=\int_{K_{j-1}}^{K_{j}} G_{j}(\tau, \mu) \psi\left(\mu, x(\mu), \mathscr{J}_{K_{j-1}^{+}}^{\Omega_{j}} x(\mu)\right) d \mu, \quad \tau \in \widetilde{J}_{j}
$$

It follows from the properties of fractional integrals and from the continuity of function $\tau^{\zeta} \psi$ that the operator $\mathscr{M}$ $: \mathscr{E}_{j} \rightarrow \mathscr{E}_{j}$ defined in (31) is well-defined. We consider the set

$$
\mathscr{B}_{R_{j}}=\left\{x \in \mathscr{E}_{j},\|x\|_{\mathscr{E}_{j}} \leq R_{j}\right\}
$$

where

$$
\begin{gathered}
R_{j}=\max \left\{\frac{3 c_{1}}{\Gamma\left(\Omega_{j}\right)}\left(\frac{K_{j}-K_{j-1}}{4}\right)^{\Omega_{j}-1}\left(\frac{K_{j}^{1-\zeta}-K_{j-1}^{1-\zeta}}{1-\zeta}\right)\right. \\
\left(\frac{3 c_{2}}{\Gamma\left(\Omega_{j}\right)}\left(\frac{K_{j}-K_{j-1}}{4}\right)^{\Omega_{j}-1}\left(\frac{K_{j}^{1-\zeta}-K_{j-1}^{1-\zeta}}{1-\zeta}\right)\right)^{1 /(1-\sigma)}, \\
\left(\frac{3 c_{3}}{\Gamma\left(\Omega_{j}\right)}\left(\frac{K_{j}-K_{j-1}}{4}\right)^{\Omega_{j}-1}\right. \\
\left.\left.\cdot\left(\frac{K_{j}^{1-\zeta}-K_{j-1}^{1-\zeta}}{1-\zeta}\right)\left(\frac{\left(K_{j}-K_{j-1}\right)^{\Omega_{j}}}{\Gamma\left(\Omega_{j}+1\right)}\right)^{\eta}\right)^{1 /(1-\eta)}\right\} .
\end{gathered}
$$

Clearly, $\mathscr{B}_{R_{j}} \neq \varnothing$ is convex, bounded, and closed. Now, we prove in the following three steps that $\mathscr{M}$ satisfies the hypotheses of Theorem 13.

Step I. $\mathscr{M}\left(\mathscr{B}_{R_{j}}\right) \subseteq\left(\mathscr{B}_{R_{j}}\right)$
For $x \in \mathscr{B}_{R_{j}}$, by Proposition 16 and (ASP2), we get

$$
\begin{aligned}
|\mathscr{M} x(\tau)|= & \left|\int_{K_{j-1}}^{K_{j}} G_{j}(\tau, \mu) \psi\left(\mu, x(\mu), \mathcal{F}_{K_{j-1}^{+}}^{\Omega_{j}} x(\mu)\right) d \mu\right| \\
\leq & \int_{K_{j-1}}^{K_{j}} G_{j}(\tau, \mu)\left|\psi\left(\mu, x(\mu), \mathcal{J}_{K_{j-1}^{+}}^{\Omega_{j}} x(\mu)\right)\right| d \mu \\
\leq & \int_{K_{j-1}}^{K_{j}} G_{j}(\tau, \mu) \mu^{-\zeta}\left(c_{1}+c_{2}|x(\mu)|^{\sigma}+c_{3}\left|\mathscr{F}_{K_{j-1}^{+}}^{\Omega_{j}} x(\mu)\right|^{\eta}\right) d \mu \\
\leq & \frac{1}{\Gamma\left(\Omega_{j}\right)}\left(\frac{K_{j}-K_{j-1}}{4}\right)^{\Omega_{j}-1} \int_{K_{j-1}}^{K_{j}} \mu^{-\zeta} \\
& \cdot\left(c_{1}+c_{2}|x(\mu)|^{\sigma}+c_{3}\left(\frac{\left(K_{j}-K_{j-1}\right)^{\Omega_{j}}}{\Gamma\left(\Omega_{j}+1\right)}\right)^{\eta}\|x\|_{\mathscr{E}_{j}}^{\eta}\right) d \mu \\
\leq & \frac{1}{\Gamma\left(\Omega_{j}\right)}\left(\frac{K_{j}-K_{j-1}}{4}\right)^{\Omega_{j}-1}\left(\frac{K_{j}^{1-\zeta}-K_{j-1}^{1-\zeta}}{1-\zeta}\right) \\
& \cdot\left(c_{1}+c_{2} R_{j}^{\sigma}+c_{3}\left(\frac{\left(K_{j}-K_{j-1}\right)^{\Omega_{j}}}{\Gamma\left(\Omega_{j}+1\right)}\right)^{\eta} R_{j}^{\eta}\right) \\
\leq & \frac{R_{j}}{3}+\frac{R_{j}}{3}+\frac{R_{j}}{3}=R_{j},
\end{aligned}
$$

which means that $\mathscr{M}\left(\mathscr{B}_{R_{j}}\right) \subseteq \mathscr{B}_{R_{j}}$.

Step II. $\mathscr{M}$ is continuous.

Assume that $\left(x_{m}\right)$ is a sequence via $x_{m} \rightarrow x$ in $\mathscr{E}_{j}$. We verify that

$$
\left\|\left(\mathscr{M} x_{m}\right)-(\mathscr{M} x)\right\|_{\mathscr{E}_{j}} \rightarrow 0, \quad m \rightarrow \infty
$$

Indeed, for $\tau \in \mathfrak{J}_{j}$, by Proposition 16 and (ASP3), we obtain

$$
\begin{aligned}
& \left|\left(\mathscr{M} x_{m}\right)(\tau)-(\mathscr{M} x)(\tau)\right| \\
& \leq \int_{K_{j-1}}^{K_{j}} G_{j}(\tau, \mu)\left|\psi\left(\mu, x_{m}(\mu), \mathcal{F}_{K_{j-1}^{+}}^{\Omega_{j}} x_{m}(\mu)\right)-\psi\left(\mu, x(\mu), \mathcal{F}_{K_{j-1}^{+}}^{\Omega_{j}} x(\mu)\right)\right| d \mu \\
& \leq \frac{1}{\Gamma\left(\Omega_{j}\right)}\left(\frac{K_{j}-K_{j-1}}{4}\right)^{\Omega_{j}-1} \int_{K_{j-1}}^{K_{j}} \mid \psi\left(\mu, x_{m}(\mu), \mathscr{F}_{K_{j-1}^{\dagger}}^{\Omega_{j}} x_{m}(\mu)\right) \\
& -\psi\left(\mu, x(\mu), \mathcal{I}_{K_{j-1}^{+}}^{\Omega_{j}} x(\mu)\right) \mid d \mu \leq \frac{1}{\Gamma\left(\Omega_{j}\right)}\left(\frac{K_{j}-K_{j-1}}{4}\right)^{\Omega_{j}-1} \\
& \cdot \int_{K_{j-1}}^{K_{j}} \mu^{-\zeta}\left(W\left|x_{m}(\mu)-x(\mu)\right|+L \mathcal{F}_{K_{j-1}^{+}}^{\Omega_{j}}\left|x_{m}(\mu)-x(\mu)\right|\right) d \mu \\
& \leq \frac{1}{\Gamma\left(\Omega_{j}\right)}\left(\frac{K_{j}-K_{j-1}}{4}\right)^{\Omega_{j}-1}\left[W\left\|x_{m}-x\right\|_{\mathscr{C}_{j}} \int_{K_{j-1}}^{K_{j}} \mu^{-\zeta} d \mu\right. \\
& \left.+L\left\|\mathscr{F}_{K_{j-1}^{\dagger}}^{\Omega_{j}}\left(x_{m}-x\right)\right\|_{\mathscr{C}_{j}} \int_{K_{j-1}}^{K_{j}} \mu^{-\zeta} d \mu\right] \\
& \leq \frac{\left(K_{j}^{1-\zeta}-K_{j-1}^{1-\zeta}\right)\left(K_{j}-K_{j-1}\right)^{\Omega_{j-1}}}{4^{\Omega_{j}-1}(1-\zeta) \Gamma\left(\Omega_{j}\right)}\left(W+\frac{L\left(K_{j}-K_{j-1}\right)^{\Omega_{j}}}{\Gamma\left(\Omega_{j}+1\right)}\right)\left\|x_{m}-x\right\|_{\mathscr{C}_{j}} .
\end{aligned}
$$

So

$$
\left\|\left(\mathscr{M} x_{m}\right)-(\mathscr{M} x)\right\|_{\mathscr{E}_{j}} \rightarrow 0 \text { as } m \rightarrow \infty
$$

Consequently, $\mathscr{M}$ is a continuous operator on $\mathscr{E}_{j}$. Step III. $\mathscr{M}$ is compact. 
Now, we will prove that $\mathscr{M}\left(\mathscr{B}_{R_{j}}\right)$ is relatively compact, meaning that $\mathscr{M}$ is compact. Clearly, $\mathscr{M}\left(\mathscr{B}_{R_{j}}\right)$ is uniformly bounded because by Step II, we have

$$
\mathscr{M}\left(\mathscr{B}_{R_{j}}\right)=\left\{\mathscr{M}(x): x \in \mathscr{B}_{R_{j}}\right\} \subset \mathscr{B}_{R_{j}} .
$$

Thus, for each $x \in \mathscr{B}_{R_{j}}$, we have $\|\mathscr{M}(x)\|_{\mathscr{C}_{j}} \leq R_{j}$ which means that $\mathscr{M}\left(\mathscr{B}_{R_{j}}\right)$ is uniformly bounded. It remains to prove that $\mathscr{M}\left(\mathscr{B}_{R_{\mathrm{j}}}\right)$ is equicontinuous.

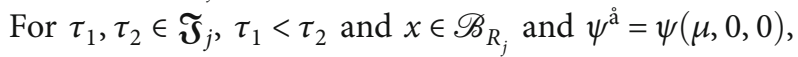
we have

$$
\begin{aligned}
& \left|(\mathscr{M} x)\left(\tau_{2}\right)-(\mathscr{M} x)\left(\tau_{1}\right)\right| \\
& =\mid \int_{K_{j-1}}^{K_{j}} G_{j}\left(\tau_{2}, \mu\right) \psi\left(\mu, x(\mu), \mathcal{F}_{K_{j-1}^{\dagger}}^{\Omega_{j}} x(\mu)\right) d \mu \\
& -\int_{K_{j-1}}^{K_{j}} G_{j}\left(\tau_{1}, \mu\right) \psi\left(\mu, x(\mu), \mathcal{F}_{K_{j-1}}^{\Omega_{j}} x(\mu)\right) d \mu \\
& \leq \int_{K_{k_{-1}}}^{K_{j}}\left|\left(G_{j}\left(\tau_{2}, \mu\right)-G_{j}\left(\tau_{1}, \mu\right)\right) \psi\left(\mu, x(\mu), \mathcal{F}_{K_{j-1}^{+}}^{\Omega_{j}} x(\mu)\right)\right| d \mu \\
& \leq \int_{K_{j-1}}^{K_{j}}\left|G_{j}\left(\tau_{2}, \mu\right)-G_{j}\left(\tau_{1}, \mu\right)\right| \mid \psi\left(\mu, x(\mu), \mathcal{F}_{K_{j-1}^{j}}^{\Omega_{j}} x(\mu)\right) \\
& -\psi(\mu, 0,0)+\psi(\mu, 0,0) \mid d \mu \\
& \leq \int_{K_{j-1}}^{K_{j}}\left|G_{j}\left(\tau_{2}, \mu\right)-G_{j}\left(\tau_{1}, \mu\right)\right| \mid \psi\left(\mu, x(\mu), \mathcal{F}_{K_{j-1}^{j}}^{\Omega_{j}} x(\mu)\right) \\
& -\psi(\mu, 0,0)\left|d \mu+\int_{K_{j-1}}^{K_{j}}\right| G_{j}\left(\tau_{2}, \mu\right)-G_{j}\left(\tau_{1}, \mu\right)|| \psi(\mu, 0,0) \mid d \mu \\
& \leq \int_{K_{j-1}}^{K_{j}}\left|G_{j}\left(\tau_{2}, \mu\right)-G_{j}\left(\tau_{1}, \mu\right)\right|\left[\mu^{-\zeta}\left(W|x(\mu)|+L\left|\mathscr{F}_{K_{j-1}}^{\Omega_{j}}(x(\mu))\right|\right)\right] d \mu \\
& +\psi^{\star} \int_{K_{j-1}}^{K_{j}}\left|G_{j}\left(\tau_{2}, \mu\right)-G_{j}\left(\tau_{1}, \mu\right)\right| d \mu \\
& \leq \int_{K_{j-1}}^{K_{j}}\left|G_{j}\left(\tau_{2}, \mu\right)-G_{j}\left(\tau_{1}, \mu\right)\right|\left[\mu^{-\zeta}\right. \\
& \left.\cdot\left(W|x(\mu)|+\frac{L\left(K_{j}-K_{j-1}\right)^{\Omega_{j}}}{\Gamma\left(\Omega_{j}+1\right)}|x(\mu)|\right)\right] d \mu+\psi^{*} \int_{K_{j-1}}^{K_{j}} \mid G_{j}\left(\tau_{2}, \mu\right) \\
& -G_{j}\left(\tau_{1}, \mu\right)\left|d \mu \leq K_{j-1}^{-\zeta}\left(W+\frac{L\left(K_{j}-K_{j-1}\right)^{\Omega_{j}}}{\Gamma\left(\Omega_{j}+1\right)}\right) R_{j} \int_{K_{j-1}}^{K_{j}}\right| G_{j}\left(\tau_{2}, \mu\right) \\
& -G_{j}\left(\tau_{1}, \mu\right)\left|d \mu+\psi^{\star} \int_{K_{j-1}}^{K_{j}}\right| G_{j}\left(\tau_{2}, \mu\right)-G_{j}\left(\tau_{1}, \mu\right) \mid d \mu,
\end{aligned}
$$

by the continuity of Green's function $G_{j}$. Hence,

$$
\left|(\mathscr{M} x)\left(\tau_{2}\right)-(\mathscr{M} x)\left(\tau_{1}\right)\right| \rightarrow 0
$$

as $\left|\tau_{2}-\tau_{1}\right| \rightarrow 0$, free of $x \in \mathscr{B}_{R_{j}}$. It implies that $\mathscr{M}\left(\mathscr{B}_{R_{j}}\right)$ is equicontinuous. From Steps I to III and the Arzela-Ascoli theorem, it can be concluded that $\mathscr{M}$ is completely continuous.
Now, from Theorem 13, problem (23) possesses at least a solution $\widetilde{x_{j}}$ in $\mathscr{B}_{R_{j}}$. We let

$$
x_{j}= \begin{cases}0, & \tau \in\left[0, K_{j-1}\right] \\ \tilde{x}_{j}, & \tau \in \widetilde{\Im}_{j} .\end{cases}
$$

We know that $x_{j} \in \mathscr{C}\left(\left[0, K_{j}\right], \mathbb{R}\right)$ defined by (41) satisfies the following equation:

$$
\begin{aligned}
& \frac{d^{2}}{d \tau^{2}}\left(\int_{0}^{K_{1}} \frac{(\tau-\mu)^{1-\Omega_{1}}}{\Gamma\left(2-\mu_{1}\right)} x_{j}(\mu) d \mu+\cdots+\int_{K_{j-1}}^{\tau} \frac{(\tau-\mu)^{1-\Omega_{j}}}{\Gamma\left(2-\Omega_{j}\right)} x_{j}(\mu) d \mu\right) \\
& \quad+\psi\left(\mu, \tilde{x}_{j}(\mu), \mathscr{F}_{0^{+}}^{\Omega_{j}} \tilde{x}_{j}(\mu)\right)=0,
\end{aligned}
$$

for $\tau \in \mathfrak{J}_{j}$, which means that $x_{j}$ is a solution of (21) with $x_{j}$ $(0)=0, x_{j}\left(K_{j}\right)=\tilde{x}_{j}\left(K_{j}\right)=0$. In consequence, we figure out that the BVP (1) admits at least a solution defined by

$$
x(\tau)=\left\{\begin{array}{l}
x_{1}(\tau), \\
x_{2}(\tau)=\left\{\begin{array}{ll}
0, & \tau \in \mathfrak{J}_{1}, \\
\tilde{x}_{2}, & \tau \in \mathfrak{J}_{2}
\end{array} .\right. \\
\cdot \\
x_{j}(\tau)= \begin{cases}0, & \tau \in\left[0, K_{j-1}\right], \\
\tilde{x}_{j}, & \tau \in \mathfrak{J}_{j},\end{cases}
\end{array}\right.
$$

and the argument is ended.

Let us discuss our second result which is relied on the Banach-CoPrp.

Theorem 18. Suppose that (ASP1) and (ASP3) hold and if

$$
\frac{\left(K_{j}^{1-\zeta}-K_{j-1}^{1-\zeta}\right)\left(K_{j}-K_{j-1}\right)^{\Omega_{j}-1}}{4^{\Omega_{j}-1}(1-\zeta) \Gamma\left(\Omega_{j}\right)}\left(W+L \frac{\left(K_{j}-K_{j-1}\right)^{\Omega_{j}}}{\Gamma\left(\Omega_{j}+1\right)}\right)<1,
$$

then, it is found a solution uniquely for BVP (1) in $\mathscr{E}_{j}$.

Proof. Let us use the Banach-CoPrp to show that $\mathscr{M}$ defined in (31) has a unique fixed point. By Proposition 16 and ( ASP3), and for $x(\tau), y(\tau) \in \mathscr{E}_{j}$ : 


$$
\begin{aligned}
& |(\mathscr{M} x)(\tau)-(\mathscr{M} y)(\tau)| \\
& =\mid \int_{K_{j-1}}^{K_{j}} G_{j}(\tau, \mu) \psi\left(\mu, x(\mu), \mathscr{F}_{K_{j-1}^{+}}^{\Omega_{j}} x(\mu)\right) d \mu \\
& -\int_{K_{j-1}}^{K_{j}} G_{j}(\tau, \mu) \psi\left(\mu, y(\mu), \mathcal{F}_{K_{j-1}^{\dagger}}^{\Omega_{j}} y(\mu)\right) d \mu \\
& \leq \int_{K_{j-1}}^{K_{j}} G_{j}(\tau, \mu) \mid \psi\left(\mu, x(\mu), \mathcal{F}_{K_{j-1}^{j}}^{\Omega_{j}} x(\mu)\right) \\
& -\psi\left(\mu, y(\mu), \mathscr{F}_{K_{j-1}^{+}}^{\Omega_{j}} y(\mu)\right) \\
& \leq \frac{1}{\Gamma\left(\Omega_{j}\right)}\left(\frac{K_{j}-K_{j-1}}{4}\right)^{\Omega_{j}-1} \int_{K_{j-1}}^{K_{j}} \mid \psi\left(\mu, x(\mu), \mathscr{F}_{K_{j-1}^{+}}^{\Omega_{j}} x(\mu)\right) \\
& -\psi\left(\mu, y(\mu), \mathcal{F}_{K_{j-1}^{+}}^{\Omega_{j}} y(\mu)\right) \\
& \leq \frac{1}{\Gamma\left(\Omega_{j}\right)}\left(\frac{K_{j}-K_{j-1}}{4}\right)^{\Omega_{j}-1} \int_{K_{j-1}}^{K_{j}} \mu^{-\zeta}(W|x(\mu)-y(\mu)| \\
& \left.+L \mathscr{F}_{K_{j-1}^{+}}^{\Omega_{j}}|x(\mu)-y(\mu)|\right) d \mu \leq \frac{1}{\Gamma\left(\Omega_{j}\right)}\left(\frac{K_{j}-K_{j-1}}{4}\right)^{\Omega_{j}-1} \\
& \cdot\left[W\|x-y\|_{\mathscr{C}_{j}} \int_{K_{j-1}}^{K_{j}} \mu^{-\zeta} d \mu+L \frac{\left(K_{j}-K_{j-1}\right)^{\Omega_{j}}}{\Gamma\left(\Omega_{j}+1\right)}\|x-y\|_{\mathscr{C}_{j}}\right. \\
& \left.\cdot \int_{K_{j-1}}^{K_{j}} \mu^{-\zeta} d \mu\right] \leq \frac{\left(K_{j}{ }^{1-\zeta}-K_{j-1}{ }^{1-\zeta}\right)\left(K_{j}-K_{j-1}\right)^{\Omega_{j-1}-1}}{4^{\Omega_{j}-1}(1-\zeta) \Gamma\left(\Omega_{j}\right)} \\
& \cdot\left(W+L \frac{\left(K_{j}-K_{j-1}\right)^{\Omega_{j}}}{\Gamma\left(\Omega_{j}+1\right)}\right)\|x-y\|_{\mathscr{E}_{j}} \cdot
\end{aligned}
$$

Consequently, by (44), the operator $\mathscr{M}$ is a contraction. Thus, by Banach-CoPrp, one can find a fixed-point $\widetilde{x_{j}} \in \mathscr{E}_{j}$ for $\mathscr{M}$ uniquely, which is the same unique solution of the BVP (23). We let

$$
x_{j}= \begin{cases}0, & \tau \in\left[0, K_{j-1}\right] \\ \tilde{x}_{j}, & \tau \in \widetilde{J}_{j} .\end{cases}
$$

By assuming $\mathscr{C}\left(\left[0, K_{j}\right], \mathbb{R}\right)$ as the set of all continuous functions from $\left[0, K_{j}\right]$ into $\mathbb{R}$, we know that $x_{j} \in \mathscr{C}\left(\left[0, K_{j}\right]\right.$, $\mathbb{R})$ defined by (46) satisfies the following equation:

$$
\begin{aligned}
& \frac{d^{2}}{d \tau^{2}}\left(\int_{0}^{K_{1}} \frac{(\tau-\mu)^{1-\Omega_{1}}}{\Gamma\left(2-\Omega_{1}\right)} x_{j}(\mu) d \mu+\cdots+\int_{K_{j-1}}^{\tau} \frac{(\tau-\mu)^{1-\Omega_{j}}}{\Gamma\left(2-\Omega_{j}\right)} x_{j}(\mu) d \mu\right) \\
& \quad+\psi\left(\mu, \tilde{x}_{j}(\mu), \mathscr{F}_{0^{+}}^{\Omega_{j}} \tilde{x}_{j}(\mu)\right)=0,
\end{aligned}
$$

for $\tau \in \mathfrak{J}_{j}$, which means that $x_{j}$ is a unique solution of (21) with $x_{j}(0)=0$ and $x_{j}\left(K_{j}\right)=\tilde{x}_{j}\left(K_{j}\right)=0$. Then,

$$
x(\tau)=\left\{\begin{array}{l}
x_{1}(\tau), \\
x_{2}(\tau)= \begin{cases}0, & \tau \in \mathfrak{\Im}_{1}, \\
\tilde{x}_{2}, & \tau \in \mathfrak{J}_{2},\end{cases} \\
\cdot \\
x_{j}(\tau)=\begin{array}{ll}
0, & \tau \in\left[0, K_{j-1}\right], \\
\tilde{x}_{j}, & \tau \in \mathfrak{\Im}_{j}
\end{array}
\end{array}\right.
$$

is one and only one solution to the proposed BVP (1) and the argument is ended.

\section{Example}

An illustrative numerical example is given in this section to apply and validate all our theoretical results.

Example 2. Consider the fractional BVP:

$$
\begin{cases}\mathfrak{D}_{0^{+}}^{\Omega(\tau)} x(\tau)+\frac{\tau^{-1 / 5}}{1+|x(\tau)|+\left|\mathcal{F}_{0^{+}}^{\Omega(\tau)} x(\tau)\right|}=0, & \tau \in \mathfrak{J}:=[0,2], \\ x(0)=0, & x(2)=0 .\end{cases}
$$

Let

$$
\psi(\tau, s, h)=\frac{\tau^{-1 / 5}}{1+|s|+|h|},(\tau, s, h) \in[0,2] \times[0,+\infty) \times[0,+\infty) ;
$$

$$
\Omega(\tau)= \begin{cases}1.7, & \tau \in \mathfrak{J}_{1}:=[0,1], \\ 1.8, & \tau \in \mathfrak{J}_{2}:=[1,2] .\end{cases}
$$

We see that $\Omega(\tau)$ satisfies condition (ASP1). We have

$$
\begin{aligned}
\tau^{1 / 5} & \left|\psi\left(\tau, s_{1}, h_{1}\right)-\psi\left(\tau, s_{2}, h_{2}\right)\right| \\
& =\left|\tau^{1 / 5}\left(\frac{\tau^{-1 / 5}}{1+\left|s_{1}\right|+\left|h_{1}\right|}-\frac{\tau^{-1 / 5}}{1+\left|s_{2}\right|+\left|h_{2}\right|}\right)\right| \\
& =\left|\frac{\left|s_{2}\right|+\left|h_{2}\right|-\left|s_{1}\right|-\left|h_{1}\right|}{\left(1+\left|s_{1}\right|+\left|h_{1}\right|\right)\left(1+\left|s_{2}\right|+\left|h_{2}\right|\right)}\right| \\
& \leq|| s_{2}|-| s_{1}||+|| h_{2}|-| h_{1}|| \leq\left|s_{1}-s_{2}\right|+\left|h_{1}-h_{2}\right| .
\end{aligned}
$$

Thus, (ASP3) holds with $\zeta=1 / 5$ and $W=L=1$. By (5), the equation of problem (49) is divided into two expressions as follows:

$$
\begin{cases}\mathfrak{D}_{0^{+}}^{1.7} x(\tau)+\frac{\tau^{-1 / 5}}{1+|x(\tau)|+\left|\mathscr{F}_{0^{+}}^{1.7} x(\tau)\right|}=0, & \tau \in[0,1], \\ \mathfrak{D}_{1^{+}}^{1.8} x(\tau)+\frac{\tau^{-1 / 5}}{1+|x(\tau)|+\left|\mathscr{F}_{0^{+}}^{1.8} x(\tau)\right|}=0, & \tau \in[1,2] .\end{cases}
$$


For $\tau \in[0,1]$, the BVP (49) is corresponding to the following BVP:

$$
\begin{cases}\mathfrak{D}_{0^{+}}^{1.7} x(\tau)+\frac{\tau^{-1 / 5}}{1+|x(\tau)|+\left|\mathscr{I}_{0^{+}}^{1.7} x(\tau)\right|}=0, & \tau \in[0,1], \\ x(0)=0, & x(1)=0 .\end{cases}
$$

We shall check that condition (44) is satisfied as follows:

$$
\begin{aligned}
& \frac{\left(K_{1}^{1-\zeta}-K_{0}{ }^{1-\zeta}\right)\left(K_{1}-K_{0}\right)^{\Omega_{1}-1}}{4^{\Omega_{1}-1}(1-\zeta) \Gamma\left(\Omega_{1}\right)}\left(W+L \frac{\left(K_{1}-K_{0}\right)^{\Omega_{1}}}{\Gamma\left(\Omega_{1}+1\right)}\right) \\
& =\frac{5}{4^{1.7} \Gamma(1.7)}\left(1+\frac{1}{\Gamma(2.7)}\right) \simeq 0.8587<1 .
\end{aligned}
$$

By Theorem 18 , it is found a solution $x_{1} \in \mathscr{E}_{1}$ uniquely to the BVP (54).

For $\tau \in[1,2]$, problem (49) can be written as follows:

$$
\begin{cases}\mathfrak{D}_{1^{+}}^{1.8} x(\tau)+\frac{\tau^{-1 / 5}}{1+|x(\tau)|+\left|\mathscr{I}_{0^{+}}^{1.8} x(\tau)\right|}=0, & \tau \in[1,2], \\ x(1)=0, & x(2)=0 .\end{cases}
$$

We see that

$$
\begin{gathered}
\frac{\left(K_{2}{ }^{1-\zeta}-K_{1}{ }^{1-\zeta}\right)\left(K_{2}-K_{1}\right)^{\Omega_{2}-1}}{4^{\Omega_{2}-1}(1-\zeta) \Gamma\left(\Omega_{2}\right)}\left(W+L \frac{\left(K_{2}-K_{1}\right)^{\Omega_{2}}}{\Gamma\left(\Omega_{2}+1\right)}\right) \\
=\frac{5\left(2^{4 / 5}-1\right)}{4^{1.8} \Gamma(1.8)}\left(1+\frac{1}{\Gamma(2.8)}\right) \simeq 0.5237<1 .
\end{gathered}
$$

Thus, condition (44) is satisfied. Therefore, by Theorem 18 , it is found a solution $\tilde{x}_{2} \in \mathscr{E}_{2}$ uniquely to the BVP (56). It is known that

$$
x_{2}(\tau)= \begin{cases}0, & \tau \in[0,1] \\ \tilde{x}_{2}(\tau), & \tau \in[1,2]\end{cases}
$$

Now, from Definition 14, the BVP (49) has a unique solution defined by

$$
x(\tau)= \begin{cases}x_{1}(\tau), & \tau \in[0,1], \\ x_{2}(\tau)= \begin{cases}0, & \tau \in[0,1], \\ \tilde{x}_{2}(\tau), & \tau \in[1,2] .\end{cases} \end{cases}
$$

\section{Conclusion}

Our proposed multiterm BVP has been successfully investigated in this work via two fixed-point theorems: Schauder-
FixPThm and Banach-CoPrp to prove the solutions' existence and uniqueness for NnLFDEqs. A numerical example is given at the end to support and validate the potentiality of all our obtained results. Therefore, all results in this work show a great potential to be applied in various applications of multidisciplinary sciences. Further investigations on this open research problem can be also possible with the help of our original results in this research paper. In other words, one can extend the proposed BVP to other complicated real mathematical fractional models by terms of newly introduced operators with nonsingular kernels in the future.

\section{Data Availability}

No data were used to support this study.

\section{Conflicts of Interest}

The authors declare that they have no competing interests.

\section{Authors' Contributions}

The authors declare that the study was realized in collaboration with equal responsibility. All authors read and approved the final manuscript.

\section{Acknowledgments}

The second and fourth authors were supported by Azarbaijan Shahid Madani University.

\section{References}

[1] I. Podlubny, Fractional Differential Equations, Academic Press, New York, USA, 1999.

[2] A. A. Kilbas, H. M. Srivastava, and J. J. Trujillo, "Theory and applications of fractional differential equations," in North-Holland Mathematics Studies, vol. 204, Elsevier, Amsterdam, 2006.

[3] D. Baleanu, S. Etemad, and S. Rezapour, "A hybrid Caputo fractional modeling for thermostat with hybrid boundary value conditions," Boundary Value Problems, vol. 2020, no. 1, 2020.

[4] H. Aydi, M. Jleli, and B. Samet, "On positive solutions for a fractional thermostat model with a convex-concave source term via $\backslash(\backslash p s i \backslash)$-Caputo fractional derivative," Mediterranean Journal of Mathematics, vol. 17, no. 1, p. 16, 2020.

[5] S. T. M. Thabet, S. Etemad, and S. Rezapour, "On a coupled Caputo conformable system of pantograph problems," Turkish Journal of Mathematics, vol. 45, no. 1, pp. 496-519, 2021.

[6] S. A. Khan, K. Shah, G. Zaman, and F. Jarad, "Existence theory and numerical solutions to smoking model under CaputoFabrizio fractional derivative," Chaos: An Interdisciplinary Journal of Nonlinear Science, vol. 29, no. 1, article 013128, 2019.

[7] S. T. M. Thabet, S. Etemad, and S. Rezapour, "On a new structure of the pantograph inclusion problem in the Caputo conformable setting," Boundary Value Problems, vol. 2020, no. 1, 2020.

[8] H. Mohammadi, S. Kumar, S. Rezapour, and S. Etemad, "A theoretical study of the Caputo-Fabrizio fractional modeling 
for hearing loss due to mumps virus with optimal control," Chaos, Solitons \& Fractals, vol. 144, p. 110668, 2021.

[9] S. Rezapour, S. Etemad, and H. Mohammadi, "A mathematical analysis of a system of Caputo-Fabrizio fractional differential equations for the anthrax disease model in animals," Advances in Difference Equations, vol. 2020, no. 1, 2020.

[10] E. Karapinar, A. Fulga, M. Rashid, L. Shahid, and H. Aydi, "Large contractions on quasi-metric spaces with an application to nonlinear fractional differential equations," Mathematics, vol. 7, no. 5, p. 444, 2019.

[11] D. Baleanu, A. Jajarmi, H. Mohammadi, and S. Rezapour, “A new study on the mathematical modelling of human liver with Caputo-Fabrizio fractional derivative," Chaos, Solitons \& Fractals, vol. 134, p. 109705, 2020.

[12] B. Alqahtani, H. Aydi, E. Karapinar, and V. Rakocevic, "A solution for Volterra fractional integral equations by hybrid contractions," Mathematics, vol. 7, no. 8, p. 694, 2019.

[13] A. K. Golmankhane, K. K. Ali, R. Yilmazer, and M. K. A. Kaabar, "Economic models involving time fractal," Journal of Mathematics and Modeling in Finance, vol. 2020, no. 1, pp. 181-200, 2020.

[14] M. M. Matar, M. I. Abbas, J. Alzabut, M. K. A. Kaabar, S. Etemad, and S. Rezapour, "Investigation of the p-Laplacian nonperiodic nonlinear boundary value problem via generalized Caputo fractional derivatives," Advances in Difference Equations, vol. 2021, no. 1, 2021.

[15] S. Rezapour, A. Imran, A. Hussain, F. Martínez, S. Etemad, and M. K. A. Kaabar, "Condensing functions and approximate endpoint criterion for the existence analysis of quantum integro-difference FBVPs," Symmetry, vol. 13, no. 3, p. 469, 2021.

[16] F. Martnez, I. Martnez, M. K. A. Kaabar, and S. Paredes, "New results on complex conformable integral," AIMS Mathematics, vol. 5, no. 6, pp. 7695-7710, 2020.

[17] F. Martnez, I. Martnez, M. K. A. Kaabar, R. Ortz-Munuera, and S. Paredes, "Note on the conformable fractional derivatives and integrals of complex-valued functions of a real variable," IAENG International Journal of Applied Mathematics, vol. 50, pp. 609-615, 2020.

[18] F. Martínez, I. Martínez, M. K. A. Kaabar, and S. Paredes, “On conformable Laplace's equation," Mathematical Problems in Engineering, vol. 2021, Article ID 5514535, 10 pages, 2021.

[19] A. Akgül, M. Inc, and D. Baleanu, "On solutions of variableorder fractional differential equations," An International Journal of Optimization and Control: Theories \& Applications (IJOCTA), vol. 7, no. 1, pp. 112-116, 2017.

[20] H. G. Sun, A. Chang, Y. Zhang, and W. Chen, "A review on variable-order fractional differential equations: mathematical, foundations, physical models, and its applications," Fractional Calculus and Applied Analysis, vol. 22, no. 1, 2018.

[21] A. Raminia, A. F. Dizaji, and V. J. Majd, "Solution existence for non-autonomous variable-order fractional differential equations," Mathematical and Computer Modelling, vol. 55, no. 34, pp. 1106-1117, 2012.

[22] S. G. Samko, "Fractional integration and differentiation of variable order," Analysis Mathematica, vol. 21, no. 3, pp. 213-236, 1995.

[23] D. Valerio and J. S. Costa, "Variable-order fractional derivatives and their numerical approximations," Signal Processing, vol. 91, no. 3, pp. 470-483, 2011.
[24] S. Zhang, S. Sun, and L. Hu, "Approximate solutions to initial value problem for differential equation of variable order," Journal of Fractional Calculus and Applications, vol. 9, no. 2, pp. 93-112, 2018.

[25] C. F. M. Coimbra, "Mechanics with variable-order differential operators," Annalen der Physik, vol. 12, no. 1112, pp. 692-703, 2003.

[26] R. Lin, F. Liu, V. Anh, and I. Turner, "Stability and convergence of a new explicit finite-difference approximation for the variable-order nonlinear fractional diffusion equation," Applied Mathematics and Computation, vol. 212, no. 2, pp. 435-445, 2009.

[27] P. Zhuang, F. Liu, V. Anh, and I. Turner, "Numerical methods for the variable-order fractional advection-diffusion equation with a nonlinear source term," SIAM Journal on Numerical Analysis, vol. 47, no. 3, pp. 1760-1781, 2009.

[28] J. F. G. Aguilar, "Analytical and numerical solutions of a nonlinear alcoholism model via variable-order fractional differential equations," Physica A, vol. 494, pp. 52-75, 2018.

[29] J. V. D. C. Sousa and E. C. de Oliverira, "Two new fractional derivatives of variable order with non-singular kernel and fractional differential equation," Computational and Applied Mathematics, vol. 37, no. 4, pp. 5375-5394, 2018.

[30] H. Zhang, S. Li, and L. Hu, "The existeness and uniqueness result of solutions to initial value problems of nonlinear diffusion equations involving with the conformable variable derivative," Revista de la Real Academia de Ciencias Exactas, Físicas y Naturales. Serie A. Matemáticas, vol. 113, no. 2, pp. 16011623, 2019.

[31] S. Zhang and L. Hu, "Unique existence result of approximate solution to initial value problem for fractional differential equation of variable order involving the derivative arguments on the half-axis," Mathematics, vol. 7, no. 286, pp. 1-23, 2019.

[32] S. Zhang, "Existence of solutions for two point boundary value problems with singular differential equations of variable order," Electronic Journal of Differential Equations, vol. 245, pp. 1-16, 2013.

[33] D. Tavares, R. Almeida, and D. F. M. Torres, "Caputo derivatives of fractional variable order numerical approximations," Communications in Nonlinear Science and Numerical Simulation, vol. 35, p. 6987, 2016.

[34] S. Zhang and L. Hu, "The existence of solutions and generalized Lyapunov-type inequalities to boundary value problems of differential equations of variable order," AIMS Mathematics, vol. 5, no. 4, pp. 2923-2943, 2020.

[35] S. G. Samko and B. Boss, "Integration and differentiation to a variable fractional order," Integral Transforms and Special Functions, vol. 1, no. 4, pp. 277-300, 1993.

[36] A. Granas and J. Dugundji, Fixed Point Theory, Springer-Verlag, New York, 2003. 